\title{
Breast Cancer in Jamaica: Stage, Grade and Molecular Subtype Distributions Across Age Blocks, the Implications for Screening and Treatment
}

\author{
Jason Copeland ${ }^{\mathrm{a}, \mathrm{b}, \mathrm{c}}$, Abimbola Oyedeji ${ }^{\mathrm{b}}$, Neggoshane Powell ${ }^{\mathrm{b}}$, Cherian J. Cherian ${ }^{\mathrm{b}, \mathrm{c}}$,

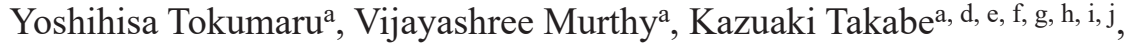 \\ Jessica Young ${ }^{\text {a }}$
}

\begin{abstract}
Background: Breast cancer is the most commonly diagnosed and leading cause of cancer-related morbidity and mortality in females worldwide. Significant disparities exist in breast cancer incidence and mortalities between low- to middle- and high-income countries. The purpose of this study was to analyze the distribution of prognostic and predictive clinicopathological features of invasive breast cancer at a single institution in Jamaica across three age groups.

Methods: Data from patients diagnosed with invasive breast cancer who underwent definitive surgery between August 2017 and September 2018 were identified. The patients were divided into three age groups $(<50,50-59$ and $>59$ years) and the distribution of tumor size, grade, molecular subtype, nodal status and anatomic stage were determined and compared with the US population registry. Comparisons of the various characteristics were performed using the Fisher's exact test.
\end{abstract}

Manuscript submitted May 14, 2021, accepted June 21, 2021

Published online July 10, 2021

aDepartment of Surgical Oncology, Roswell Park Comprehensive Cancer Center, Buffalo, NY 14263, USA

bepartment of General Surgery, Kingston Public Hospital, Kingston, Jamaica, WI

${ }^{\mathrm{c}}$ Department of Surgery, Anesthesia, Radiology and Emergency Medicine, University of West Indies, Mona, Jamaica, WI

dDepartment of Gastroenterological Surgery, Yokohama City University Graduate School of Medicine, Yokohama 236-0004, Japan

eDepartment of Surgical Oncology, Graduate School of Medicine, Gifu University, 1-1 Yanagido, Gifu 501-1194, Japan

fDepartment of Gastrointestinal Tract Surgery, Fukushima Medical University School of Medicine, Fukushima 960-1295, Japan

gDepartment of Surgery, Jacobs School of Medicine and Biomedical Sciences, State University of New York, Buffalo, NY 14263, USA

${ }^{h}$ Department of Surgery, Niigata University Graduate School of Medical and Dental Sciences, Niigata 951-8510, Japan

iDepartment of Breast Surgery and Oncology, Tokyo Medical University, Tokyo 160-8402, Japan

${ }^{j}$ Corresponding Author: Kazuaki Takabe, Roswell Park Cancer Institute, Elm and Carlton Streets, Buffalo, NY 14623, USA.

Email: kazuaki.takabe@roswellpark.org

doi: https://doi.org/10.14740/wjon1389
Results: Ninety-nine definitive operations were performed and met the criteria for analysis. Average age at the time of diagnosis was 54 years compared to 62 years reported in the US databases. Thirty-six percent of the patients presented below age 50 years, which was twice the corresponding rate reported for Caucasian females $(18 \%)$ in the USA. Fifty percent of patients in our registry had axillary lymph node metastases at presentation and they were younger than patients with negative axillary nodes (95\% confidence interval (CI) -12.06 to -1.93 , $\mathrm{P}=0.007)$. Patients in the age group less than age 50 years were more likely to have advanced stage, high histological grade cancers compared to the older age blocks $(95 \%$ CI $0.039-0.902, \mathrm{P}=0.033)$.

Conclusion: Invasive breast cancer presents at an earlier age in Jamaican women and is associated with poor prognostic features such as high rates of axillary lymph node metastases, high histological grade, advanced stage, triple-negative subtypes and low luminal A subtypes.

Keywords: Breast cancer disparities; Breast cancer in Jamaica; Racial disparities; Breast cancer; Low-income countries

\section{Introduction}

Breast cancer is the most common cancer affecting women worldwide and is the leading cause of cancer-related morbidity and mortality in Caribbean females [1-5]. Although the trends in breast cancer incidence and mortality have been decreasing in high-income countries, this is in contrast to middle-and low-income countries with increasing corresponding trends over the past few decades $[4,5]$. As a region, despite having a moderate age standardized incidence rate of 50.2/100,000 cases/people, the Caribbean has the fourth highest breast cancer mortality rate in the world [6]. Analysis of the prognostic and predictive factors of invasive breast cancer in individual lowto middle-income countries allows for better understanding of needs of breast cancer management and the targeted application of limited resources in these countries.

In the context of individualized approach to breast cancer management, it is no longer enough to assess overall population data, but local heterogeneity within populations should be 
KPH-R

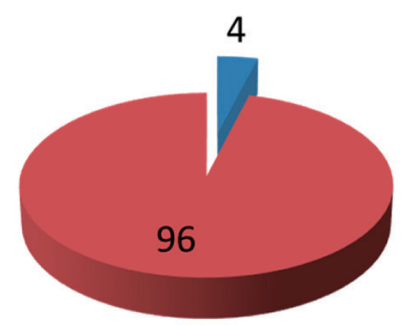

SEER

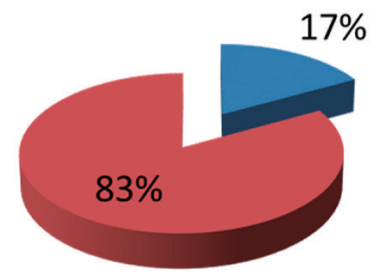

\section{DCIS \\ Invasive}

Figure 1. Pie chart representing the distribution of new breast cancers in percentages between invasive and ductal carcinoma in situ (DCIS) in the KPH-R over 14-month period under study and USA using the SEER database (2012 - 2015). SEER: Surveillance Epidemiology and End Result; KPH-R: Kingston Public Hospital Breast Cancer Registry.

analyzed as well. One of these areas of heterogeneity relates to the distribution of invasive breast cancer biology across age groups. Breast cancer in younger patients have often been found to be associated with more aggressive behavior, advanced disease at presentation and poorer overall survival particularly in low- and middle-income countries [7-9]. Conversely, through associations such as the Society of Surgical Oncology, breast cancer in the elderly has been purported to have more favorable outcomes with their choosing wisely guidelines, recommending de-escalation of axillary lymph node surgery in elderly patients with favorable invasive breast cancer biology [10].

We aimed to retrospectively analyze a prospectively maintained intra-department breast cancer registry database at the Kingston Public Hospital (KPH) to determine the age, grade, stage and molecular features of breast cancer in our population. Despite there being no consensus definition for age limits that separate young and old patients with invasive breast cancer, we divided our patients into age blocks less than 50, 50 - 59 and > 59 years due to the possible practical implications such as in breast cancer screening recommendations.

\section{Materials and Methods}

The Kingston Public Hospital Breast Cancer Registry (KPH$\mathrm{R}$ ) is a prospectively collected database of all patients consecutively diagnosed with breast cancer who had definitive surgery with curative or palliative intent. Following waiver from the Institutional Review Board and Research Ethics, the database was retrospectively assessed, and the corresponding medical records were retrieved. The following patient information variables were abstracted and analyzed by the lead investigators: age at presentation, gender, tumor size, tumor grade, axillary lymph node status, molecular subtype and overall anatomic stage. Staging was assigned using the American Joint Committee on Cancer (AJCC) seventh edition manual [11].

The primary objective was to determine the differences in distribution of the abstracted patient information variables across predetermined age blocks $(<50,50-59$ and $>59$ years $)$.
We included all patients regardless of the completeness of the patient's data parameters and where any of the parameters was absent, we assigned an "unknown" category. This allowed for more complete assessment of the registry, including the data deficit rate. Patients with in situ disease or recurrent disease in ipsilateral breast were excluded, as were patients with nonepithelial cancers. The American Cancer Society (ACS) 2011 - 2016 and the Surveillance, Epidemiology and End Results (SEER) 2013 - 2017 databases were used as a comparative framework for the US data in our analysis [12]. Statistical analysis was done using the SPSS software and the Fisher's exact test was applied to test for statistical significance and all tests were two-sided. A P-value $<0.05$ was considered statistically significant.

\section{Results}

Of the 103 definitive operations for primary breast cancer that were performed between August 2017 and September 2018 at the KPH, four (4\%) were for pure ductal carcinoma in situ (DCIS) and 99 (96\%) were for primary invasive breast cancers. The average age of presentation for patients with DCIS was 57 years. These patients were excluded from the final analysis. Of the 99 patients with primary invasive carcinoma, 96 (97\%) were females and three (3\%) were males. Comparative analysis of the ACS 2019 published estimates for invasive breast cancer, where they estimated over 260,000 new cases of invasive breast, showed overall DCIS rate of $18 \%$ and only $1 \%$ of invasive cancers being diagnosed in men and the SEER database with 17\% DCIS and 83\% invasive cancers (Fig. 1).

\section{Age and nodal distribution}

The average age of presentation for patients with primary invasive breast cancer in the KPH-R was 54 years for females and 67 years for males, while the mean overall age at presentation for females in the ACS data was 62 years with African American women presenting at a slightly earlier age (60 years) 
Table 1. Distribution of the Grades of New Breast Cancers in the SEER Database (2012 - 2015) and KPH-R (2017 - 2018)

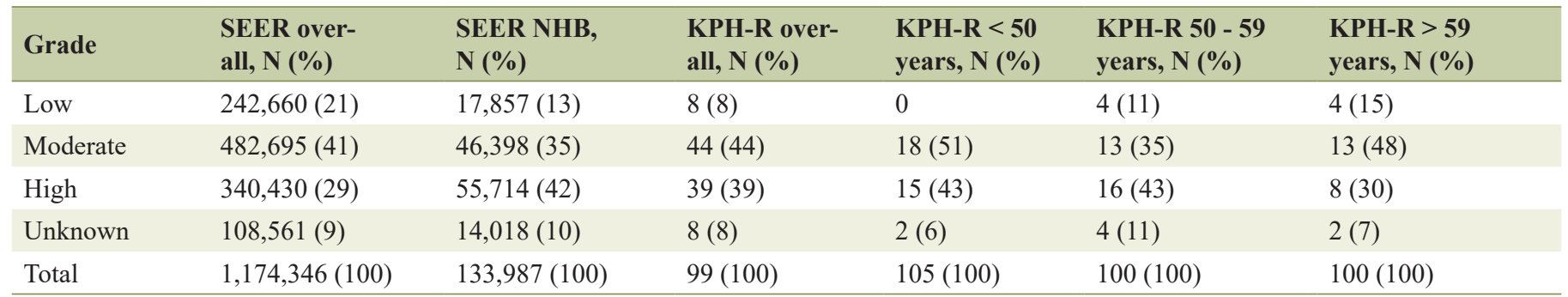

The overall grades are reported for the SEER, SEER NHB and KPH-R along with grades across age groups in the KPH-R. NHB denotes African American patients. SEER: Surveillance Epidemiology and End Result; KPH-R: Kingston Public Hospital Breast Cancer Registry; NHB: non-Hispanic Black.

than non-Hispanic Caucasian women (63 years). Our findings therefore reflect much earlier ages of presentation for invasive breast cancers in the KPH-R cohort compared with both the non-Hispanic Caucasians and the African American patients in the ACS database. We found that $36 \%$ of our patients were in the age group $<50$ years, and this was twice the corresponding rate reported in the ACS database (18\%). Patients in the age block 50 - 59 years accounted for $37 \%$ and only $27 \%$ were older than 59 years in the KPH-R (Fig. 1).

The presence of axillary lymph node metastases was determined on final pathology with hematoxylin and eosin staining and was unknown in $24 \%$ of patients in the KPH-R. However, for those with known statuses, half of them were node-positive and the other half were node-negative. The mean age of presentation for node-positive patients was 50 years, while node negative patients were older with mean of 57 years $(95 \%$ confidence interval (CI) -12.06 to $-1.93, \mathrm{P}=0.007)$. The nodal positivity rates for patients in the age blocks $<50$ and $>59$ years were $49 \%$ and $26 \%$, respectively $(95 \%$ CI -1.41 to 43.34 , $\mathrm{P}=0.07)$. The corresponding rate for patients aged $50-59$ years was $38 \%$. The rates for positive axillary lymph nodes in the ACS database were reported as $26 \%$ for non-Hispanic Caucasians and 33\% for African American women, both much lower than the rates seen in our cohort.

\section{Grade and stage distribution}

Histological grade was assessed using the Nottingham modification of the Scarff-Bloom-Richardson system. Overall only $8 \%$ of patients in this study presented with low-grade (grade 1 ) invasive breast cancer while intermediate (grades 2) and high (grade 3) grades accounted for $43 \%$ and $40 \%$, respectively (95\% CI 23.33-45.58\%, P $<0.0001)$. When analyzed across age blocks, we noted that none of the patients in age block $<50$ years presented with low-grade invasive breast cancer, whereas $43 \%$ had high grade (95\% CI 25.12-59.28\%, P < 0.0001).
In the age block $>59$ years, $15 \%$ of patients presented with low-grade cancer and $30 \%$ presented with high-grade invasive breast cancer. The age block 50 - 59 years had similar highgrade disease rates $(44 \%)$ to the age group below 50 years, but only $11 \%$ of patients presented with low-grade invasive breast cancer (Table 1). Comparatively, $21 \%$ and $29 \%$ of patients in the overall SEER database had low- and high-grade cancers at diagnosis, respectively.

There were no noticeable differences in the stages of invasive breast cancer at presentation among the three age blocks in the KPH-R. Overall $9 \%$ of our patients presented with stage I disease while stages II and III accounted for $41 \%$ and $40 \%$, respectively, with $10 \%$ of patients having undetermined stage. The rate of stage III breast cancer in the age group $<50$ years was the highest at $51 \%$. In the ACS breast cancer database, localized invasive breast cancer accounted for $66 \%$ of nonHispanic Caucasian females and 56\% in non-Hispanic African American females.

We analyzed a sub-group deemed to be "extremely aggressive breast cancer" based on advanced anatomic stage with high histological grade at presentation (stage III with grade 3 ). This represented $17 \%$ of the overall population of patients under study (Table 2). Patients less than 50 years old were significantly over-represented in this sub-group accounting for $65 \%$, while the age blocks $50-59$ and $>59$ years accounted for three patients (18\%) each (95\% CI 0.039 - 0.902 , $\mathrm{P}=0.033$ ).

\section{Molecular subtype distribution}

The molecular subtypes of invasive breast cancers in the KPH-R were determined by standard immunohistochemistry. Patients were divided into four main groups: luminal A (hormone receptor $(\mathrm{HR})+$ /human epidermal growth factor receptor 2 (HER2)-), luminal B (HR+/HER2+), HER2 over-expressed (HR-/HER2+) and triple-negative (HR-/HER2-). HR positiv-

Table 2. Distribution of Patients With Stage III Invasive Breast Cancer With High Histological Grade Across Age Groups in KPH-R

\begin{tabular}{lllll} 
& $<\mathbf{5 0}$ years & $\mathbf{5 0}-\mathbf{5 9}$ years & $>\mathbf{5 9}$ years & P value \\
\hline Stage III with grade 3, N (\%) & $11(65)$ & $3(18)$ & $3(18)$ & 0.033 \\
\hline
\end{tabular}

KPH-R: Kingston Public Hospital Breast Cancer Registry. 


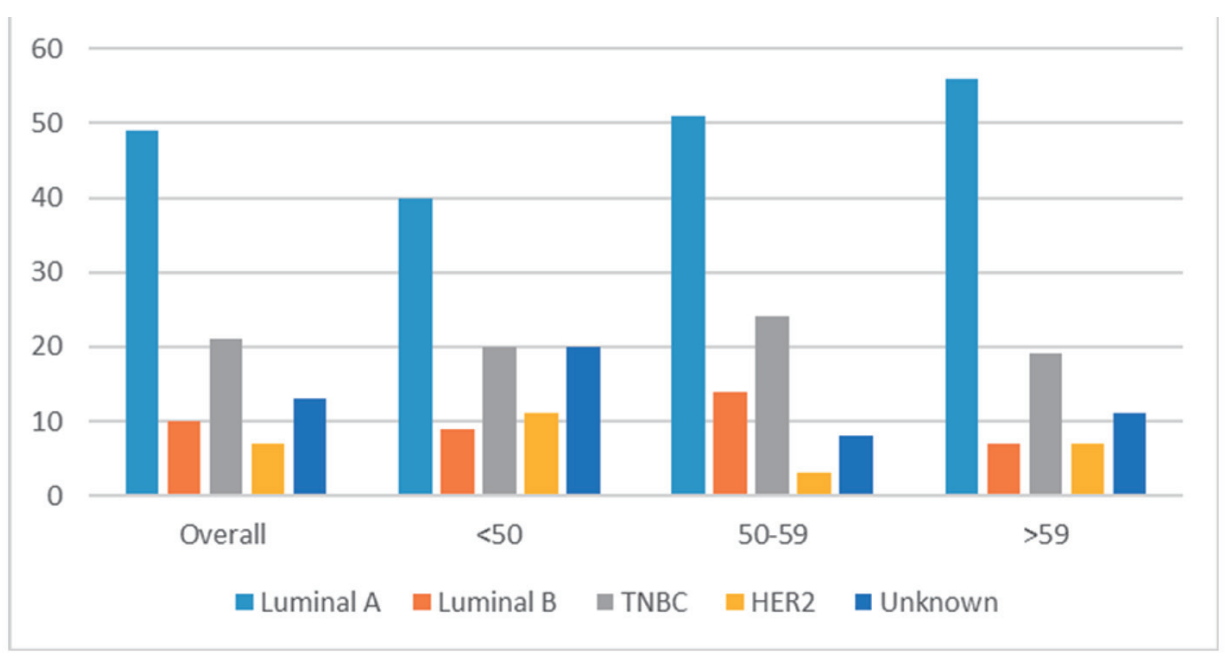

Figure 2. Bar chart depicting the distribution of new breast cancers (in percentages) by cancer subtype for patients. Overall < 50 years, 50 - 59 years, > 59 years in the KPH-R over the 14-month period under study. KPH-R: Kingston Public Hospital Breast Cancer Registry.

ity accounted for $59 \%$ of the entire population (49\% luminal A and $10 \%$ luminal B) with a mean age of 56, while HR-negative patients had a mean of 52 years (not statistically significant) (Fig. 2). Two major differences were identified between the molecular subtypes from the KPH-R and the ACS databases. Firstly, the luminal A subtype rate of $49 \%$ in the KPH-R was well below the overall ACS rate of $73 \%$ (76\% for non-Hispanic Caucasians and 61\% for non-Hispanic African Americans). The luminal B subtype rates were comparable between the KPH-R (10\%) and the ACS (11\%) databases. The second major discrepancy was that the triple-negative subtype rate accounted for $21 \%$ of all patients in the KPH-R, almost twice the documented rate for non-Hispanic Caucasian females in the ACS database, though it was identical to the rate found in non-Hispanic African American. The HER2 positivity subtype accounted for $17 \%$ in the KPH-R (HR-/HER2+ plus $\mathrm{HR}+/$ HER2+) which was similar to the $15 \%$ across the ACS and
SEER database (Figs. 2-6).

There were no differences in the triple-negative molecular subtypes across the pre-determined age blocks. The rate of luminal A-positive subtype in age block $<50$ years was $40 \%$, while the rate was $56 \%$ in the oldest age block ( $>59$ years) $(95 \% \mathrm{CI}-8.58 \%$ to $38.15 \%, \mathrm{P}=0.21)$. There were no other appreciable differences among the age blocks and the unknown rate for molecular subtype was $13 \%$ for the entire population (Table 3).

\section{Discussion}

In our analysis we were able to demonstrate that Jamaican women present at earlier ages with primary breast cancers than American women, even when compared with the African American population subset. We also demonstrated high axil-

\section{ACS (2012-2016)}

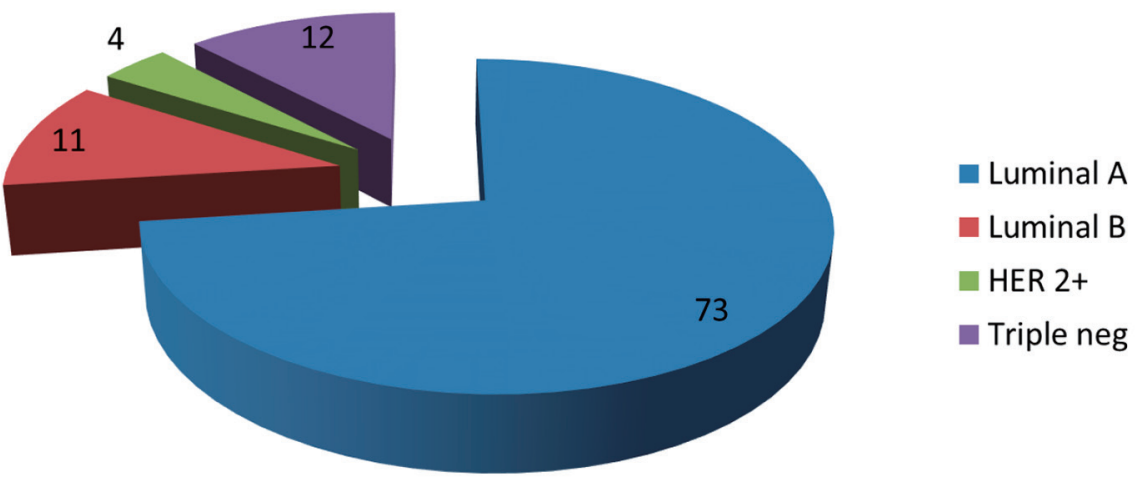

Figure 3. Pie chart depicting distribution of new invasive breast cancers (in percentages) by cancer subtype in the American Cancer Society database 2012 - 2016. 


\section{SEER - overall (2012-2015)}

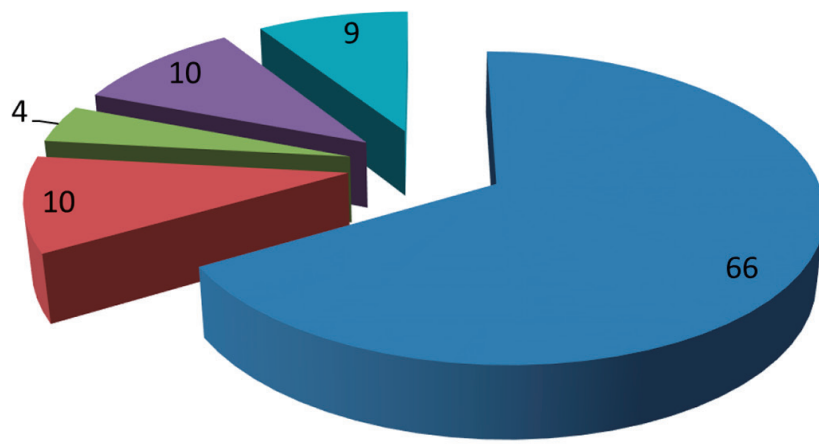

- Luminal A

- Luminal B

HER 2+

Triple neg

- Unknown

Figure 4. Pie chart depicting the distribution of new invasive breast cancers (in percentages) by cancer subtype in the SEER database 2012 - 2015. SEER: Surveillance Epidemiology and End Result.

\section{SEER Non Hispanic Blacks}

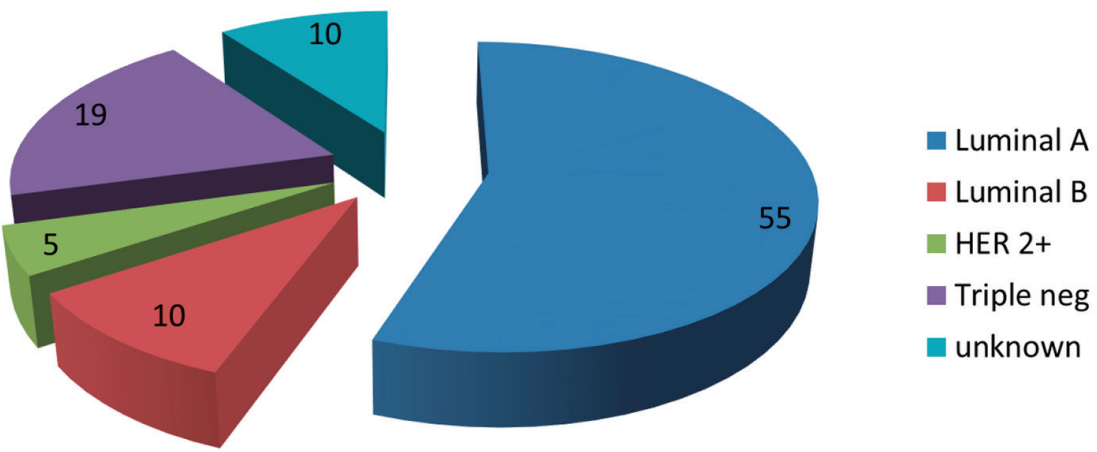

Figure 5. Pie chart depicting distribution of new breast cancers in non-Hispanic Blacks by cancer subtype in the SEER database 2012 - 2015. SEER: Surveillance Epidemiology and End Result.

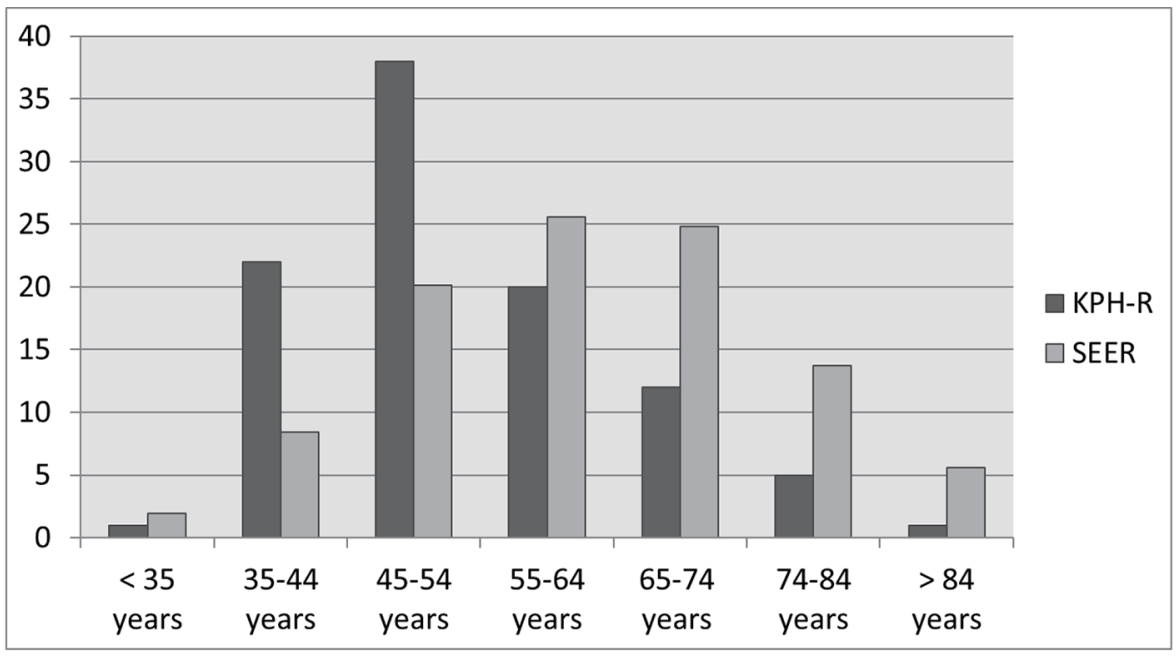

Figure 6. Bar chart depicting the age distributions of new breast cancers (in percentages) in KPH-R over the 14-month period under study and the SEER database 2012 - 2015. SEER: Surveillance Epidemiology and End Result; KPH-R: Kingston Public Hospital Breast Cancer Registry. 
Table 3. Distribution of Grade, Molecular Subtype, Tumor Size, Nodal Status and Stage Across Age Groups in the KPH-R

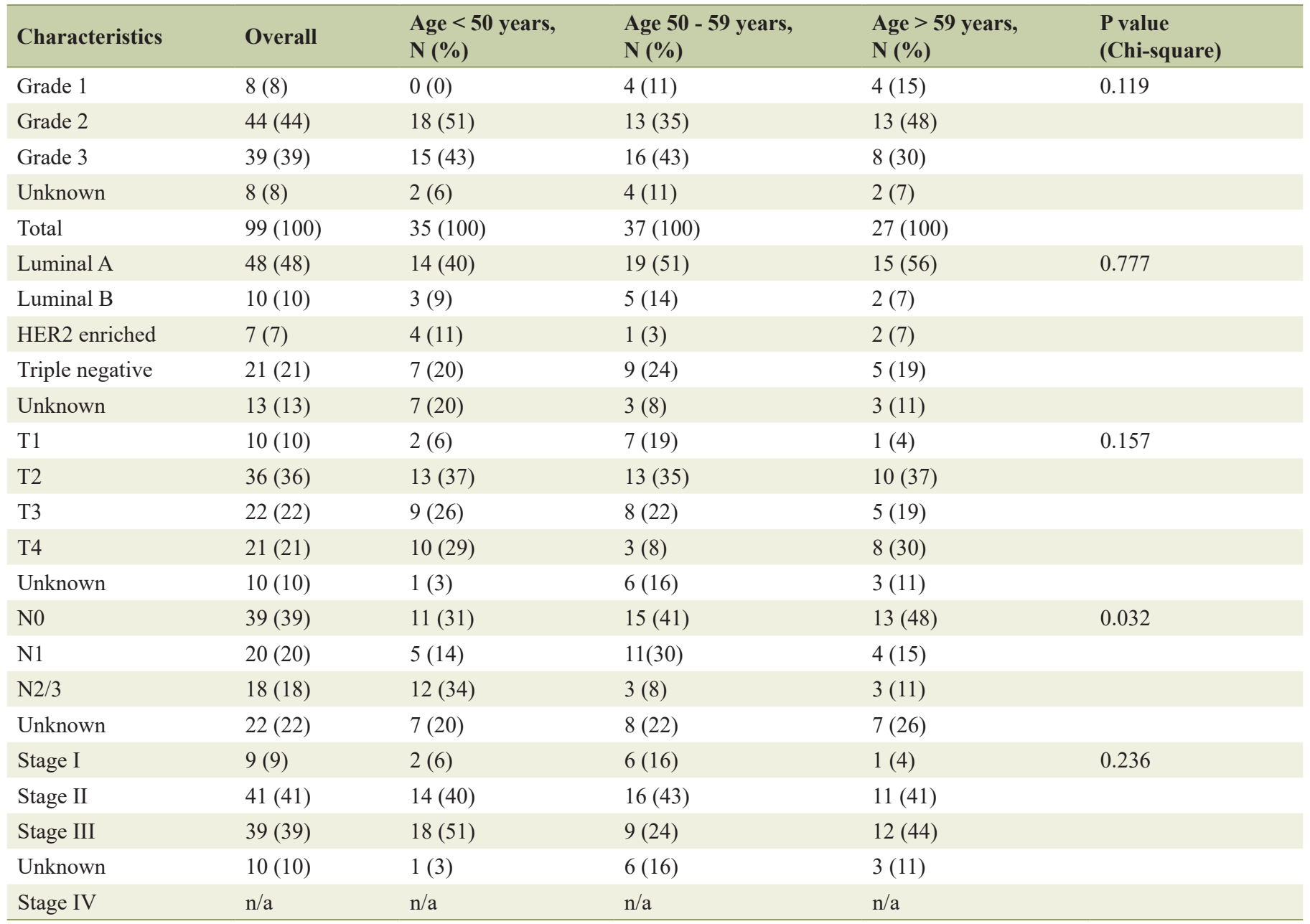

KPH-R: Kingston Public Hospital Breast Cancer Registry.

lary lymph node involvement at presentation in our patients and patients with positive axillary lymph nodes were significantly younger than patients with negative nodes. Younger patients are less likely to have favorable histological grades and presented with more advanced axillary nodal disease. These results help to explain the high mortality burden associated with the diagnosis of breast cancer in Jamaican women, especially for patients in the age group less than 50 years. It will be of interest in the future to evaluate women under 40 years and compare their results with women $<40$ years from the SEER data, in order to evaluate the significance of screening in that population.

The incidence of breast cancer continues to increase globally and disproportionately so in low- and middle-income countries such as Jamaica where the age-standardized incidence rate of breast cancer has moved from 43 per 100,000 in 2010 to 56.8 per 100,000 in 2018 [13, 14]. While highincome countries and upper middle-income countries account for approximately $75 \%$ of the global breast cancer incidence, there is significant disparity in the burden of the disease with low-income and lower middle-income countries accounting for $40 \%$ of the global breast cancer deaths [5]. The cumulative decline in breast cancer mortality in high-income countries such as the USA has been reported to be as high as $40 \%$ over the last three decades [12]. However, the trend in countries such as Jamaica and the rest of the Caribbean has been that of steady increase, which is partly related to the advanced stage of the disease at presentation [3, 15-17]. In fact, a recent study highlighting the 25-year trend in global breast cancer mortality showed that the Caribbean and Latin America have the highest increasing trend in breast cancer mortality during the years 1990 - 2015 [17].

The Jamaican population is estimated to be over 2.9 million people with life expectancy for females being 74 years and $92 \%$ of the population identifying as having some or mostly African ancestry $[18,19]$. The age-standardized mortality rate of breast cancer in Jamaican females has most recently been reported at 23.2 per 100,000 [14]. The stage distribution of breast cancer found in the KPH-R highlighted the high proportion of late stage invasive breast cancer in our population with approximately $40 \%$ of all patients presenting with stage 3 disease with the greatest burden $(51 \%)$ of women in the age block $<50$ years having this presentation. This high burden 
of disease in the younger age block was further confirmed on subgroup analysis of patients with advanced nodal disease plus high histological grade, where $65 \%$ of the subgroup were in the age block $<50$ years $(\mathrm{P}=0.03)$. These findings in our patients under age 50 years are concordant with the more aggressive behavior and worse prognosis of breast cancer in young patients that is reported in the literature [20-22].

Jamaica and the United States have similar female life expectancies, therefore the differences seen in the age distributions of breast cancer are not due to differences in their respective life expectancies [19]. The earlier mean age of presentation in the KPH-R (54 years) database against the US data (62 years) persisted even when analyzed against the subgroup of African American women (61 years) [12]. This relative leftward shift in (earlier) presentation of breast cancer is mirrored in other populations across the Caribbean, such as Barbados, and the Bahamas, and is consistent with the previous reports on the Jamaican population [23-26].

Currently, there is no national standardized breast cancer screening program in Jamaica and it has been estimated that less than $5 \%$ of Jamaican women eligible for mammographic screening actually get them [27]. In the KPH-R we found that only $23 \%$ of our patients with invasive breast cancer had any screening mammograms in the preceding 5 years, which compares poorly with the $73 \%$ rate of mammographic screening "over 2 years" reported in the ACS database [12].

From our findings the implications for screening are particularly important given that $36 \%$ of women in the KPH-R presented at age less than 50 years $(28 \%$ of patients falling the age range 40 - 49 years), which was twice as high as that reported in the SEER and ACS databases (18\%) (see figure) [12, 28]. While the American Society of Breast Surgeons (ASBrS) recommends commencing mammographic screening in average risk patients at age 40 years, the ACS has recently adjusted their guidelines to recommend commencement at 45 years and the US Preventive Services Task Force (USPSTF) recommendation is currently at 50 years with biennial intervals [2932]. Many guidelines continue to recommend mammographic breast cancer screening for patients in their $40 \mathrm{~s}$ with annual screening intervals [29, 31, 33-36]. It is also generally agreed on that annual screening is more appropriate for younger patients (premenopausal) due to the aggressive nature of breast cancer and higher rates of false-negative mammography in these patients, leading to the ACS recommendation for annual screening for patients aged 45 - 54 years $[30,37]$. Additionally, premenopausal women that are screened at intervals greater than annually 9 - 15 months have been found to be associated with later stage at presentation, increased rates of interval breast cancers and overall less favorable prognostic characteristics [38].

When the mean outcomes of six Cancer Intervention and Surveillance Modeling Network (CISNET) models looking at three different screening recommendations, in terms of benefits and risks, were analyzed, they found that mean mortality reductions were greatest for annual screening beginning at 40 years $(39.6 \%)$ compared with the hybrid ACS recommendation of annual screening from 45 to 54 years and biennially thereafter $(30.8 \%)$ or the USPSTF guideline for biennial screening beginning at age 50 years $(23.2 \%)$ [39]. The societal recom- mendations from high-income countries, although serving as important benchmark guidelines, do not specifically address patients in our population and therefore the most effective age at which to start and the interval of mammographic screening for breast cancer remains uncertain for our patients. However, with such a large proportion of our patients presenting with invasive breast cancer prior to age 50 years, the adoption of the USPSTF criteria would likely be ineffective in achieving the often cited reductions in mortality rates of $35-40 \%$ that are associated with mammographic screening [30, 39-41]. Early onset of annual mammographic screening beginning at age 40 years would be the most effective approach based on our findings, with the option of transitioning to biennial screening in the older population in whom breast cancer biology is usually less aggressive.

Triple-negative breast cancer (TNBC) is a poor prognostic feature associated with aggressive behavior, high rates of treatment failures (recurrences), higher rates of brain and visceral metastases and worse overall survival $[42,43]$. They often present at a later stage which contributes to their dismal prognosis compared to HR-positive and HER2 over-expressed invasive breast cancer. The finding of high rates of TNBC in our database was not surprising considering the well-documented high TNBC rates in patients of African descent and the high rate of African ancestry makeup of the Jamaican population [44, 45]. Our TNBC rates are similar to the African American population, as reported in the ACS database, and to other populations of patients in the Caribbean with invasive breast cancer [12, $16,44,46,47]$. The response of TNBC to chemotherapy is generally better than that of HR-positive breast cancers with pathological complete response (pCR) rates ranging from $22 \%$ to $52 \%[48,49]$. The addition of immunotherapy to chemotherapy has proven effective in improving outcomes in these patients especially if they are programmed death-ligand 1 (PDL1)-positive [50, 51]. In our population with high proportions of young patients and TNBC, immunotherapy is a promising therapeutic option for improving the poor survival outcomes that we are seeing in these patients.

Approximately $10 \%$ of breast cancers are associated with pathogenic germline genetic mutations and more than $50 \%$ of them are mutations in BRCA 1 and 2 genes [52-54]. These BRCA mutations are present in high rates in TNBCs, being five and a half times more likely than their non-triple-negative counterparts $[55,56]$. In the Caribbean, high BRCA mutation rates have been found in countries such as the Bahamas where the prevalence of these mutations was as high as $23 \%$ in women with breast cancer [57]. These high BRCA mutation rates carry significant implications for treatment as well as for genetic testing availability in our patients, with recent phase III randomized control trials and international consensus guidelines reporting prolonged survival for advanced TNBC patients having BRCA mutations that were treated with platinum-based chemotherapy and PARP-inhibitors [58, 59]. In addition, neo-adjuvant chemotherapy is increasingly being utilized in patients with operable TNBC to improve breast conservation rates, to decrease extent of axillary lymph node dissections as well as to identify incomplete responders who may derive better survival with the subsequent inclusion of capecitabine therapy $[60,61]$. 
The proportion of women with HR-positive subtypes reported in the ACS database ( $84 \%$ ) is much higher than the overall rate of $57 \%$ found in the KPH-R or in any of our analyzed age blocks. HR-positive invasive breast cancers are associated with less aggressive disease with $50 \%$ better mortality rates compared to HR-negative subtypes [62]. With that in mind, there are currently options for de-escalation of chemotherapy in HR-positive patients with uninvolved axillary lymph nodes with favorable genomic scores $[63,64]$. However, the much lower rates of HR positivity as well as high positive nodal disease burden of $50 \%$ mean less of our patients would be good candidates for this practice of chemotherapy omission or for other practices such as decreasing axillary lymph node dissections or consideration for the omission of whole breast radiation therapy post breast conservative surgery [65-69]. In fact, the high rates of stage 3 breast cancer in the Jamaican population $(40 \%)$ argue for greater access to chest wall and regional nodal radiation to improve loco-regional disease control and mortality rates $[70,71]$.

The highest rates for estrogen receptor positivity in our patients were demonstrated in age block $>59$ years at $63 \%$, but it was only $49 \%$ for patients in the $<50$ years age block. This trend of lower HR positivity is also reported in the African American population where younger women especially have more HR-negative breast cancer subtypes $[72,73]$. The higher rates of HR positivity seen with advancing age blocks are well established in the literature and the generally less aggressive nature of breast cancer in the elderly population is reflective of this subtype [74-76].

The histological grading of invasive breast cancer is an important prognostic factor which maintains its significance irrespective of the status of the axillary lymph nodes and as a result grading has been incorporated into the new AJCC eighth edition of the breast cancer staging manual [77-80]. Women in the KPH-R were less likely to have low-grade invasive breast cancers $(8 \%)$ compared to high grade $(43 \%)$ which is consistent with studies from Nigeria and elsewhere where proportions of aggressive behaving breast cancers, in women of African descent, as high as $79 \%$ are reported $[81,82]$.

Approximately $15-20 \%$ of patients with invasive breast cancers typically over-express HER2 which are associated with aggressive clinical behavior and poor outcomes in the absence of systemic therapy with various HER2 targeted therapies such as trastuzumab, pertuzumab, tyrosine kinase inhibitors or antibody-drug conjugates [83-86]. As noted in Table $1,17 \%$ of Jamaican women had HER2-positive breast cancer and would benefit from targeted therapies, but these drug treatment options remain prohibitively expensive for most Jamaican women.

\section{Conclusion}

Invasive breast cancer presents at an earlier average age in Jamaican women compared to the US population and it is associated with poor prognostic features such as higher rates of axillary node positivity, advanced stage, high histological grades, and low luminal A and high triple negative subtypes particularly in younger women. Low- to middle-income countries such as ours with high and increasing breast cancer case fatality rates need to aggressively formulate and implement nationally coordinated early detection, screening and treatment protocols that are based on our local or regional data. Emphasis must be placed on early age of onset of mammographic screening and targeted resource utilization in an effort to reverse the trends of increasing breast cancer morbidity and mortality in our populations.

\section{Limitations}

This study is limited by its size and the issues associated with incomplete data retrieval which averaged approximately $10 \%$ across all examined variables. The inability to fully stage patients with advanced imaging modalities prevented us from ascribing an accurate anatomic stage 4 in the KPH-R. Despite these limitations, this paper is unique as we believe it is the first attempt to examine clinicopathological and immunohistological variables for invasive breast cancer across age stratification in Jamaican women.

\section{Acknowledgments}

We acknowledge Dr. Lindberg Simpson and Dr. Natalie Whylie for institutional support and Mr. Gooden for data retrieval.

\section{Financial Disclosure}

This work was supported by US National Institutes of Health/ National Cancer Institute grant 5T32CA108456 to VM, R01CA160688, R01CA250412, R37CA248018, US Department of Defense BCRP grant W81XWH-19-1-0674, as well as the Edward K. Duch Foundation and Paul \& Helen Ellis Charitable Trust to KT, and US National Cancer Institute cancer center support grant P30-CA016056 to Roswell Park Comprehensive Cancer Center.

\section{Conflict of Interest}

The authors have no conflict of interest to declare.

\section{Informed Consent}

Not applicable.

\section{Author Contributions}

Conceptualization: JC, KT. Methodology: JC, NP, AO. Formal analysis: JC, KT, JY, VM, YT, CC. Writing original draft preparation: JC. Writing review and editing: KT, VM. Supervision: KT. Project administration: KT. All authors have read and agreed to the published version of the manuscript. 


\section{Data Availability}

The data supporting the findings of this study are available from the corresponding author upon reasonable request.

\section{References}

1. Servick K. Breast cancer. Breast cancer: a world of differences. Science. 2014;343(6178):1452-1453.

2. DeSantis CE, Bray F, Ferlay J, Lortet-Tieulent J, Anderson $\mathrm{BO}$, Jemal A. International variation in female breast cancer incidence and mortality rates. Cancer Epidemiol Biomarkers Prev. 2015;24(10):1495-1506.

3. Razzaghi H, Quesnel-Crooks S, Sherman R, Joseph R, Kohler B, Andall-Brereton G, Ivey MA, et al. Leading causes of cancer mortality - Caribbean region, 2003-2013. MMWR Morb Mortal Wkly Rep. 2016;65(49):1395-1400.

4. Bray F, Ferlay J, Soerjomataram I, Siegel RL, Torre LA, Jemal A. Global cancer statistics 2018: GLOBOCAN estimates of incidence and mortality worldwide for 36 cancers in 185 countries. CA Cancer J Clin. 2018;68(6):394424.

5. Bellanger M, Zeinomar N, Tehranifar P, Terry MB. Are global breast cancer incidence and mortality patterns related to country-specific economic development and prevention strategies? J Glob Oncol. 2018;4:1-16.

6. Bray F, Pineros M. Cancer patterns, trends and projections in Latin America and the Caribbean: a global context. Salud Publica Mex. 2016;58(2):104-117.

7. Maggard MA, O'Connell JB, Lane KE, Liu JH, Etzioni DA, Ko CY. Do young breast cancer patients have worse outcomes? J Surg Res. 2003;113(1):109-113.

8. Chen HL, Zhou MQ, Tian W, Meng KX, He HF. Effect of age on breast cancer patient prognoses: a populationbased study using the SEER 18 database. PLoS One. 2016;11(10):e0165409.

9. El Saghir NS, Seoud M, Khalil MK, Charafeddine M, Salem ZK, Geara FB, Shamseddine AI. Effects of young age at presentation on survival in breast cancer. BMC Cancer. 2006;6:194.

10. Society of Surgical Oncology. Five things physicians and patients should question. Choosing Wisely. 2016.

11. Edge S, Byrd D, Compton C. AJCC cancer staging handbook from the AJCC cancer staging manual. 7th Ed Chicago, IL: Springer; 2010.

12. American Cancer Society. Breast cancer facts \& figures 2019-2020. Atlanta: American Cancer Society, Inc. 2019.

13. Gibson TN, Hanchard B, Waugh N, McNaughton D. Agespecific incidence of cancer in Kingston and St. Andrew, Jamaica, 2003-2007. West Indian Med J. 2010;59(5):456464.

14. Global Cancer Observatory. International association of cancer registries c/o international agency for research on cancer 2019.

15. Naraynsingh V, Hariharan S, Dan D, Bhola S, Bhola $\mathrm{S}$, Nagee K. Trends in breast cancer mortality in Trinidad and Tobago - a 35-year study. Cancer Epidemiol.
2010;34(1):20-23.

16. Ragin C, Banydeen R, Zhang C, Ben A, Calabrese V, Villa NN, Reville J, et al. Breast cancer research in the caribbean: analysis of reports from 1975 to 2017. J Glob Oncol. 2018;4:1-21.

17. Azamjah N, Soltan-Zadeh Y, Zayeri F. Global trend of breast cancer mortality rate: a 25-year study. Asian Pac J Cancer Prev. 2019;20(7):2015-2020.

18. United Nations Department of Economic and Social affairs, Population Division. World Population Prospects. 2019. Vol. 1 Comprehensive Tables.

19. CIA World Factbook. 2018.

20. Assi HA, Khoury KE, Dbouk H, Khalil LE, Mouhieddine TH, El Saghir NS. Epidemiology and prognosis of breast cancer in young women. J Thorac Dis. 2013;5(Suppl 1):S2-8.

21. Khambri D, Harahap W, Rustam R. Prognosis of very young women (35 years) with operable breast cancer in Padang-Indonesia. The Breast 2018;41(1):S3.

22. Elkum N, Dermime S, Ajarim D, Al-Zahrani A, Alsayed A, Tulbah A, Al Malik O, et al. Being 40 or younger is an independent risk factor for relapse in operable breast cancer patients: the Saudi Arabia experience. BMC Cancer. 2007;7:222.

23. Shirley SE, Sinclair PA, Stennett MA, Codrington G, Bhatt R, Escoffery CT. The pathology of breast cancer in Jamaica: the National Public Health Laboratory study. West Indian Med J. 2010;59(2):177-181.

24. Chin SN, Green C, Strachan GG, Wharfe G. Clinicopathologic characteristics of breast cancer in Jamaica. Asian Pac J Cancer Prev. 2014;15(7):3319-3322.

25. Mungrue K, Chase H, Gordon J, Knowles D, Lockhart K, Miller N, Morley T, et al. Breast cancer in the Bahamas in 2009-2011. Breast Cancer (Auckl). 2016;10:45-52.

26. Hennis A, Hambleton I, Nemesure B. Breast Cancer incidence and mortality in a Caribbean population: comparison with African Americans. Int J Cancer 2009;124(2):429-433.

27. Soares D, Kirlew K, Johnson P, Reid M. Mammographic referral patterns for two breast imaging units in Jamaica. West Indian Med J. 2007;56(2):159-162.

28. Howlader N, Noone A, Krapcho M. SEER cancer statistics review, 1975-2016, National Cancer Institute.

29. Siu AL, Force USPST. Screening for breast cancer: U.S. preventive services task force recommendation statement. Ann Intern Med. 2016;164(4):279-296.

30. Oeffinger $\mathrm{KC}$, Fontham ET, Etzioni R, Herzig A, Michaelson JS, Shih YC, Walter LC, et al. Breast cancer screening for women at average risk: 2015 guideline update from the American Cancer Society. JAMA. 2015;314(15):1599-1614.

31. Willey S, Whitworth P, Boolbol S. American society of breast surgeons position statement on screening mammography. 2019.

32. U.S. Preventive Services Task Force. Screening for breast cancer: U.S. Preventive Services Task Force recommendation statement. Ann Intern Med. 2009;151(10):716726, W-236.

33. Gradishar WJ, Anderson BO, Balassanian R, Blair SL, 
Burstein HJ, Cyr A, Elias AD, et al. NCCN guidelines insights: breast cancer, Version 1.2017. J Natl Compr Canc Netw. 2017;15(4):433-451.

34. Fitzpatrick-Lewis D, Hodgson N, Ciliska D. Breast cancer screening. Ottawa(ON): Canadian Task Force on Preventive Health Care; 2011.

35. Lauby-Secretan B, Scoccianti C, Loomis D, BenbrahimTallaa L, Bouvard V, Bianchini F, Straif K, et al. Breastcancer screening - viewpoint of the IARC Working Group. N Engl J Med. 2015;372(24):2353-2358.

36. Practice bulletin No. 179 summary: breast cancer risk assessment and screening in average-risk women. Obstet Gynecol. 2017;130(1):241-243.

37. Myers ER, Moorman P, Gierisch JM, Havrilesky LJ, Grimm LJ, Ghate S, Davidson B, et al. Benefits and harms of breast cancer screening: a systematic review. JAMA. 2015;314(15):1615-1634.

38. Miglioretti DL, Zhu W, Kerlikowske K, Sprague BL, Onega T, Buist DS, Henderson LM, et al. Breast tumor prognostic characteristics and biennial vs annual mammography, age, and menopausal status. JAMA Oncol. 2015;1(8):1069-1077.

39. Arleo EK, Hendrick RE, Helvie MA, Sickles EA. Comparison of recommendations for screening mammography using CISNET models. Cancer. 2017;123(19):3673-3680.

40. Tabar L, Fagerberg G, Duffy SW, Day NE. The Swedish two county trial of mammographic screening for breast cancer: recent results and calculation of benefit. J Epidemiol Community Health. 1989;43(2):107-114.

41. Kerlikowske K, Grady D, Rubin SM, Sandrock C, Ernster VL. Efficacy of screening mammography. A meta-analysis. JAMA. 1995;273(2):149-154.

42. Dent R, Trudeau M, Pritchard KI, Hanna WM, Kahn HK, Sawka CA, Lickley LA, et al. Triple-negative breast cancer: clinical features and patterns of recurrence. Clin Cancer Res. 2007;13(15 Pt 1):4429-4434.

43. Goncalves H, Guerra R, Teixeira T. Survival study of triple-negative and non-triple-negative breast cancer in a Brazilian cohort. Clin Med Insights Oncol. 2018;12.

44. Stark A, Kleer CG, Martin I, Awuah B, Nsiah-Asare A, Takyi V, Braman M, et al. African ancestry and higher prevalence of triple-negative breast cancer: findings from an international study. Cancer. 2010;116(21):4926-4932.

45. Ismail-Khan R, Bui MM. A review of triple-negative breast cancer. Cancer Control. 2010;17(3):173-176.

46. Siddharth S, Sharma D. Racial disparity and triple-negative breast cancer in African-American women: a multifaceted affair between obesity, biology and socioeconomic determinants. Cancers. 2018;10(12):514.

47. Hercules SM, Hercules JC, Ansari A, Date SAJ, Skeete DHA, Smith Connell SP, Pond GR, et al. High triple-negative breast cancer prevalence and aggressive prognostic factors in Barbadian women with breast cancer. Cancer. 2020;126(10):2217-2224.

48. Masuda H, Baggerly KA, Wang Y, Zhang Y, Gonzalez-Angulo AM, Meric-Bernstam F, Valero V, et al. Differential response to neoadjuvant chemotherapy among 7 triplenegative breast cancer molecular subtypes. Clin Cancer Res. 2013;19(19):5533-5540.
49. Liedtke C, Mazouni C, Hess KR, Andre F, Tordai A, Mejia JA, Symmans WF, et al. Response to neoadjuvant therapy and long-term survival in patients with triple-negative breast cancer. J Clin Oncol. 2008;26(8):1275-1281.

50. Schmid P, Adams S, Rugo HS, Schneeweiss A, Barrios CH, Iwata H, Dieras V, et al. Atezolizumab and nab-paclitaxel in advanced triple-negative breast cancer. N Engl J Med. 2018;379(22):2108-2121.

51. Marra A, Viale G, Curigliano G. Recent advances in triple negative breast cancer: the immunotherapy era. BMC Med. 2019;17(1):90.

52. van der Groep P, van der Wall E, van Diest PJ. Pathology of hereditary breast cancer. Cell Oncol (Dordr). 2011;34(2):71-88.

53. Castera L, Krieger S, Rousselin A, Legros A, Baumann JJ, Bruet $\mathrm{O}$, Brault B, et al. Next-generation sequencing for the diagnosis of hereditary breast and ovarian cancer using genomic capture targeting multiple candidate genes. Eur J Hum Genet. 2014;22(11):1305-1313.

54. Cancer Genome Atlas, Network. Comprehensive molecular portraits of human breast tumours. Nature. 2012; 490(7418):61-70.

55. Tun NM, Villani G, Ong K, Yoe L, Bo ZM. Risk of having BRCA1 mutation in high-risk women with triple-negative breast cancer: a meta-analysis. Clin Genet. 2014;85(1):4348.

56. Shimelis H, LaDuca H, Hu C, Hart SN, Na J, Thomas A, Akinhanmi $\mathrm{M}$, et al. Triple-negative breast cancer risk genes identified by multigene hereditary cancer panel testing. J Natl Cancer Inst. 2018;110(8):855-862.

57. Akbari MR, Donenberg T, Lunn J, Curling D, Turnquest T, Krill-Jackson E, Zhang S, et al. The spectrum of BRCA1 and BRCA2 mutations in breast cancer patients in the Bahamas. Clin Genet. 2014;85(1):64-67.

58. Robson M, Im SA, Senkus E, Xu B, Domchek SM, Masuda $\mathrm{N}$, Delaloge $\mathrm{S}$, et al. Olaparib for metastatic breast cancer in patients with a germline BRCA mutation. N Engl J Med. 2017;377(6):523-533.

59. Paluch-Shimon S, Pagani O, Partridge AH, Abulkhair O, Cardoso MJ, Dent RA, Gelmon K, et al. ESO-ESMO 3rd international consensus guidelines for breast cancer in young women (BCY3). Breast. 2017;35:203-217.

60. Pilewskie M, Morrow M. Axillary nodal management following neoadjuvant chemotherapy: a review. JAMA Oncol. 2017;3(4):549-555.

61. Masuda N, Lee SJ, Ohtani S, Im YH, Lee ES, Yokota I, Kuroi K, et al. Adjuvant Capecitabine for Breast Cancer after Preoperative Chemotherapy. N Engl J Med. 2017;376(22):2147-2159.

62. Dunnwald LK, Rossing MA, Li CI. Hormone receptor status, tumor characteristics, and prognosis: a prospective cohort of breast cancer patients. Breast Cancer Res. 2007;9(1):R6.

63. Sparano JA, Gray RJ, Makower DF, Pritchard KI, Albain KS, Hayes DF, Geyer CE, Jr., et al. Adjuvant chemotherapy guided by a 21-gene expression assay in breast cancer. N Engl J Med. 2018;379(2):111-121.

64. Paik S, Shak S, Tang G, Kim C, Baker J, Cronin M, Baehner FL, et al. A multigene assay to predict recurrence of tamox- 
ifen-treated, node-negative breast cancer. N Engl J Med. 2004;351(27):2817-2826.

65. Giuliano AE, McCall L, Beitsch P, Whitworth PW, Blumencranz P, Leitch AM, Saha S, et al. Locoregional recurrence after sentinel lymph node dissection with or without axillary dissection in patients with sentinel lymph node metastases: the American College of Surgeons Oncology Group Z0011 randomized trial. Ann Surg. 2010;252(3):426-432; discussion 432-423.

66. Donker M, van Tienhoven G, Straver ME, Meijnen P, van de Velde CJ, Mansel RE, Cataliotti L, et al. Radiotherapy or surgery of the axilla after a positive sentinel node in breast cancer (EORTC 10981-22023 AMAROS): a randomised, multicentre, open-label, phase 3 non-inferiority trial. Lancet Oncol. 2014;15(12):1303-1310.

67. Kunkler IH, Williams LJ, Jack WJ, Cameron DA, Dixon JM, investigators PI. Breast-conserving surgery with or without irradiation in women aged 65 years or older with early breast cancer (PRIME II): a randomised controlled trial. Lancet Oncol. 2015;16(3):266-273.

68. Hughes KS, Schnaper LA, Berry D, Cirrincione C, McCormick B, Shank B, Wheeler J, et al. Lumpectomy plus tamoxifen with or without irradiation in women 70 years of age or older with early breast cancer. N Engl J Med. 2004;351(10):971-977.

69. Krag DN, Anderson SJ, Julian TB, Brown AM, Harlow SP, Costantino JP, Ashikaga T, et al. Sentinel-lymph-node resection compared with conventional axillary-lymphnode dissection in clinically node-negative patients with breast cancer: overall survival findings from the NSABP B-32 randomised phase 3 trial. Lancet Oncol. 2010;11(10):927-933.

70. EBCTCG (Early Breast Cancer Trialists' Collaborative Group), McGale P, Taylor C, Correa C, Cutter D, Duane F, Ewertz M, et al. Effect of radiotherapy after mastectomy and axillary surgery on 10-year recurrence and 20-year breast cancer mortality: meta-analysis of individual patient data for 8135 women in 22 randomised trials. Lancet. 2014;383(9935):2127-2135.

71. Overgaard M, Hansen PS, Overgaard J, Rose C, Andersson M, Bach F, Kjaer M, et al. Postoperative radiotherapy in high-risk premenopausal women with breast cancer who receive adjuvant chemotherapy. Danish Breast Cancer Cooperative Group 82b Trial. N Engl J Med. 1997;337(14):949955.

72. Allott EH, Geradts J, Cohen SM, Khoury T, Zirpoli GR, Bshara W, Davis W, et al. Frequency of breast cancer subtypes among African American women in the AMBER consortium. Breast Cancer Res. 2018;20(1):12.

73. Troester M, Sun X, Allott E, et al. Racial differences in PAM 50 subtypes in the Carolina breast cancer study. Journal of the National Cancer Institute. 2018;110:170-182.

74. Pourzand A, Fakhree MB, Hashemzadeh S, Halimi M,
Daryani A. Hormone receptor status in breast cancer and its relation to age and other prognostic factors. Breast Cancer (Auckl). 2011;5:87-92.

75. Sorlie T, Perou CM, Tibshirani R, Aas T, Geisler S, Johnsen $\mathrm{H}$, Hastie T, et al. Gene expression patterns of breast carcinomas distinguish tumor subclasses with clinical implications. Proc Natl Acad Sci U S A. 2001;98(19):1086910874.

76. Li Y, Yang D, Yin X, Zhang X, Huang J, Wu Y, Wang $\mathrm{M}$, et al. Clinicopathological characteristics and breast cancer-specific survival of patients with single hormone receptor-positive breast cancer. JAMA Netw Open. 2020;3(1):e1918160.

77. Schwartz AM, Henson DE, Chen D, Rajamarthandan S. Histologic grade remains a prognostic factor for breast cancer regardless of the number of positive lymph nodes and tumor size: a study of 161708 cases of breast cancer from the SEER Program. Arch Pathol Lab Med. 2014;138(8):1048-1052.

78. Bloom HJ, Richardson WW. Histological grading and prognosis in breast cancer; a study of 1409 cases of which 359 have been followed for 15 years. Br J Cancer. 1957;11(3):359-377.

79. Elston CW, Ellis IO. Pathological prognostic factors in breast cancer. I. The value of histological grade in breast cancer: experience from a large study with long-term follow-up. Histopathology. 1991;19(5):403-410.

80. Giuliano AE, Edge SB, Hortobagyi GN. Eighth Edition of the AJCC Cancer Staging Manual: Breast Cancer. Ann Surg Oncol. 2018;25(7):1783-1785.

81. Forae G, Nwachokor F, Igbe A. Histopathological profile of breast cancer in an African population. Ann Med Health Sci Res. 2014;4(3):369-373.

82. Henson DE, Chu KC, Levine PH. Histologic grade, stage, and survival in breast carcinoma: comparison of African American and Caucasian women. Cancer. 2003;98(5):908917.

83. Cronin KA, Harlan LC, Dodd KW, Abrams JS, BallardBarbash R. Population-based estimate of the prevalence of HER-2 positive breast cancer tumors for early stage patients in the US. Cancer Invest. 2010;28(9):963-968.

84. Slamon D, Eiermann W, Robert N, Pienkowski T, Martin M, Press M, Mackey J, et al. Adjuvant trastuzumab in HER2positive breast cancer. N Engl J Med. 2011;365(14):12731283.

85. von Minckwitz G, Procter M, de Azambuja E, Zardavas D, Benyunes M, Viale G, Suter T, et al. Adjuvant pertuzumab and trastuzumab in early HER2-positive breast cancer. N Engl J Med. 2017;377(2):122-131.

86. von Minckwitz G, Huang CS, Mano MS, Loibl S, Mamounas EP, Untch M, Wolmark N, et al. Trastuzumab emtansine for residual invasive HER2-positive breast cancer. N Engl J Med. 2019;380(7):617-628. 